JOURNAL OF INTEGRAL EQUATIONS

AND APPLICATIONS

Volume 3, Number 4, Fall 1991

\title{
MULTI-STEP METHODS FOR FIRST KIND SINGULAR VOLTERRA INTEGRAL EQUATIONS
}

\author{
C.J. GLADWIN AND L.E. GAREY
}

\begin{abstract}
Multi-step methods are described for first kind singular Volterra integral equations. Methods of order three are constructed and illustrated with a numerical example.
\end{abstract}

1. Introduction. Consider the linear Volterra integral equation with weakly singular kernel of the form

$$
\begin{gathered}
b y(x)+\int_{0}^{x}(x-t)^{-\alpha} K(x, t) y(t) d t=f(x), \\
0 \leq t \leq x \leq a, 0 \leq \alpha<1
\end{gathered}
$$

where $K$ and $f$ are given functions and $y$ is the unknown function to be found. The constant $b$ equals 1 or 0 corresponding to a second or first kind equation, respectively. Conditions on $K$ and $f$ which provide for a unique solution $y(x) \in C[0, a]$ may be found in Pogorzelski [17], for example.

The methods considered in Section two are applicable to all equations of the form (1.1). This is a nontrivial problem for the case $b=0$ where it has been well known for almost 20 years that a root condition must be imposed on the weights of the quadrature rule method along with the usual consistency conditions, for $\alpha=0$ even. Methods for the case $b=1$ may also be found in Brunner [2], Garey [6, 7], DeHoog and Weiss [11], and Linz [12]. Single step methods for the case $b=0$ may be found in Brunner [1], Linz [13], and Weiss and Anderssen [19].

Cameron and McKee [3] have considered multi-step methods for the case $b=0$. However, their root condition is more intractable than the one given here since they require knowledge about the roots of a power series. Lubich [14] has recently constructed methods which are based on having a multi-step method $(\rho, \sigma)$ of order $p$ with both the

The research of the second author was supported by NSERC of Canada under grant A8196.

Copyright (C)1991 Rocky Mountain Mathematics Consortium 
polynomials $\rho$ and $\sigma$ satisfying a simple von Neumann condition. It is well known that such methods exist for $p \leq 6$ (backward differentiation or Adams-Bashforth). His methods are silent for $p>6$ despite his unproven statement in $[\mathbf{1 4}$, p. 100].

The methods discussed in this paper may be treated as a generalization of those of Weiss [18] or Gladwin [8]. Furthermore, the root condition given here can still be used to construct methods of higher order as in Gladwin [9] and [10] for $\alpha=0$.

We close this section with an existence theorem for (1.1) in the case $b=0$.

Theorem 1. In equation (1.1), with $b=0$, assume

(i) $K, K_{x} \in C\left[S_{2}\right], S_{2}=\{(x, t) \mid 0 \leq t \leq x \leq a\}$,

(ii) $K(x, x) \neq 0$ on $S_{1}=\{x \mid 0 \leq x \leq a\}$,

(iii) $F(x)=\int_{0}^{x} f(t)(x-t)^{\alpha-1} d t$,

(iv) $0<\alpha<1$.

Then, a necessary and sufficient condition for (1.1) with $b=0$ to have $a$ unique continuous solution, $y(x)$, is that $f(0)=0$ and $F(x) \in C^{1}\left(S_{1}\right)$.

Proof. See Pogorzelski $[\mathbf{1 7}$, p. 16]. The theorem uses the Dirichlet transformation to remove the singularity in (1.1) and hence reduces it to a nonsingular equation. Thus, with sufficient smoothness on $K(x, t)$ and $f(x)$, and $f^{j}(0)=0, j=0,1, \ldots, r$ (where $r$ is the degree of smoothness, one can have a sufficiently smooth solution $y(x)$.

2. Multi-step methods. Let $I_{N}=\left\{x_{n}=n h, n=0(1) N, h>\right.$ $0, N h=a\}$ denote a partition of $[0, a]$ and write equation (1.1) with $b=0$ as

$$
\begin{aligned}
\int_{0}^{x_{p}}\left(x_{n}-t\right)^{-\alpha} & K\left(x_{n}, t\right) y(t) d t \\
& +\sum_{j=p+1}^{n} \int_{x_{j-1}}^{x_{j}}\left(x_{n-t}\right)^{-\alpha} K\left(x_{n}, t\right) y(t) d t=f\left(x_{n}\right)
\end{aligned}
$$

where $p$ is a given nonnegative integer. Using appropriate quadrature rules for the integrals in equation (2.1), a class of $p+1$ step methods 
are obtained which may be used to generate approximations $y_{n}$ to $y\left(x_{n}\right), n=p(1) N$. For multi-step methods, $(p>0)$, starting values $y_{n} n=0(1) p-1$ must be generated initially by some independent method such as a single step method $[\mathbf{1}, \mathbf{1 9}]$. Note that

$$
y(0)=y_{0}=\lim _{x \rightarrow 0}(1-\alpha) f(x) x^{\alpha-1} / K(0,0)
$$

which gives $y(0)=0$. These latter methods could be used to generate the entire solution, but they typically increase the number of evaluations of the kernel, $K$, by a factor of order $r^{2}$ where $r$ is the order of the method.

An appropriate set of quadrature rules can be obtained as follows:

i) for each $n$, replace $K\left(x_{n}, t\right) y(t)$ on $\left[0, x_{p}\right]$ by a polynomial in $\pi_{p}$ which interpolates $K\left(x_{n}, t\right) y(t)$ at the points $t=x_{i}, i=0(1) p$

ii) similarly, replace $K\left(x_{n}, t\right) y(t)$ on each interval $\left[x_{j-1}, x_{j}\right], j=$ $p+1(1) N$, by a polynomial in $\pi_{q_{j}}\left(q_{j} \leq p+1\right)$ which interpolates at the points $t=x_{j-i}, i=0(1) q_{j}$. This leads to a method of the form

$$
\begin{aligned}
& \sum_{i=0}^{p} \gamma_{n, i}(\alpha) K\left(x_{n}, x_{i}\right) y_{i} \\
& +\sum_{j=p+1}^{n} \sum_{i=0}^{q_{j}} \beta_{(n-j) i}(\alpha) K\left(x_{n}, x_{j-i}\right) y_{j-i}=h^{\alpha-1} f\left(x_{n}\right), \\
& n=p(1) N
\end{aligned}
$$

where the required starting values may be determined by a single step method. The coefficients $\gamma_{n, i}(\alpha)$ and $\beta_{(n-j) i}(\alpha)$ may be determined for a given $\alpha$ by the "method of undetermined coefficients." The resulting systems of linear equations are summarized in the following lemmas. In these lemmas it is assumed that $g$ is a sufficiently smooth function.

Lemma 1. Let

$$
E_{n, p}(g)=\int_{0}^{x_{p}}\left(x_{n}-t\right)^{-\alpha} g(t) d t-h^{1-\alpha} \sum_{i=0}^{p} \gamma_{n, i}(\alpha) g\left(x_{i}\right)
$$

Then

$$
E_{n, p}(g)=C_{n, p} h^{p+2-\alpha} g^{(p+1)}(\xi), \quad \xi \in\left(0, x_{p}\right)
$$


if and only if

$$
\begin{aligned}
\sum_{i=0}^{p} \gamma_{n, i}(\alpha)(n-i)^{\ell} & =\left[n^{\ell+1-\alpha}-(n-p)^{\ell+1-\alpha}\right] /(\ell+1-\alpha) \\
\ell & =0(1) p, \quad n=p(1) N, \quad\left(C_{n, p}, \text { constant }\right) .
\end{aligned}
$$

Proof. The details may be found in Gladwin [8]. The result is easily obtained by replacing $g(t)$ by $\left(x_{n}-t\right)^{\ell}, \ell=0(1) p$ in equation (2.3).

Lemma 2. Let

$$
E_{n, j}(g)=\int_{x_{j-1}}^{x_{j}}\left(x_{n}-t\right)^{-\alpha} g(t) d t-h^{1-\alpha} \sum_{i=0}^{q_{j}} \beta_{(n-j) i}(\alpha) g\left(x_{j-i}\right)
$$

Then $E_{n, j}(g)=D_{n, j} h^{q_{j}+2-\alpha} g^{\left(q_{j}+1\right)}\left(\eta_{j}\right), \eta_{j} \in\left(x_{j-1}, x_{j}\right)$ if and only if

$$
\begin{gathered}
\sum_{i=0}^{q_{j}} \beta_{(n-j) i}(\alpha)(n-j+i)^{\ell} \\
=\left[(n-j+1)^{\ell+1-\alpha}-(n-j)^{\ell+1-\alpha}\right] /(\ell+1-\alpha) \\
\ell=0(1) q_{j}, \quad j=p+1(1) n, \quad\left(D_{n, j}, \text { constant }\right) .
\end{gathered}
$$

Proof. As in lemma one, the result follows easily by replacing $g(t)$ by $\left(x_{n}-t\right)^{\ell}, \ell=0(1) q_{j}$ in equation (2.4). The details may be found in Gladwin [8].

3. Convergence results. For ease of notation, the argument $(\alpha)$ shall be omitted for $\gamma_{n i}$ and $\beta_{(n-j) i}$ from now on. The polynomials

$$
\sigma_{j}(z)=\sum_{i=0}^{q_{j}} \beta_{j, i} z^{q_{j}-i} j=0(1) n-(p+1)
$$

are called the characteristic polynomials of the method (2.2). In the following we shall also need the reciprocal polynomials

$$
\sigma_{j}^{*}(z)=z^{q_{j}} \sigma\left(z^{-1}\right)=\sum_{i=0}^{q_{j}} \beta_{j, i} z^{i} .
$$


The method (2.2) with $K(x, t) \equiv 1$ together with the starting values may be viewed as a system of $N+1$ linear equations

$$
A_{N} Y_{N}=B_{N}
$$

where $A_{N}$ is a partitioned matrix of order $N+1$

$$
A_{N}=\left[\begin{array}{cc}
D_{\gamma} & O \\
\Gamma & W_{N}
\end{array}\right]
$$

with $D_{\gamma}: d_{i j}=\delta_{i j}$, Kronecker delta, $i=0(1) p-1, j=0(1) p$

$$
d_{p j}=\gamma_{p, j}, \quad j=0(1) p
$$

$O$ : rectangular zero matrix,

$\Gamma$ : rectangular matrix depending on $\gamma_{n, j}, n>p$,

$Y_{N}:\left(y_{0}, \ldots, y_{N}\right)^{T}$,

$B_{N}:\left(y_{0}, \ldots, y_{p-1}, h^{\alpha-1} f\left(x_{p}\right), \ldots, h^{\alpha-1} f\left(x_{n}\right)\right)^{T}$

The $(N-p) \times(N-p)$ matrix $W_{N}$, which depends only on the $\beta_{(n-j) i}$, is an $m(=1)$ block isoclinal lower triangular matrix. See, also, McKee [15] where a general discussion of properties concerning the existence of uniform bounds for the inverses of such matrices may be found.

For ease of notation, let $W_{N}=\left(w_{i j}\right), i, j=0(1) N-p-1$.

Lemma 3. Assume

1) $\gamma_{n, i}$ and $\beta_{(n-j) i}$ are as determined in lemmas 1 and 2 with $q_{j}=p+1$ for all $j$

2) $\sigma_{0}(z)$ is a simple von Neumann polynomial, i.e., all the roots lie within $|z| \leq 1$ with only simple roots on the boundary

3) $0 \leq \alpha<1$.

Then $\|\Gamma\|_{\infty}<\infty,\left\|W_{N}\right\|_{\infty}<\infty$ and $\left\|W_{N}^{-1}\right\|_{\infty}<\infty$.

Proof. The first part follows by a simple but tedious inversion of the Vandermonde matrix in lemma 2 and observing assumption 3. As in McKee [15], the generating function of $W$ is

$$
g(W, z)=\sum_{i=0}^{\infty} w_{i, 0} z^{i}
$$


At this point we consider the explicit dependence of the $W$ on $\beta_{n-j, i}$ alluded to above. In equation (2.2) with $K(x, t) \equiv 1$ and $q_{j}=p+1$ for all $j$, we need to consider

$$
\begin{aligned}
\sum_{j=p+1}^{n} \sum_{i=0}^{p+1} \beta_{n-j, i} y_{j-i}= & \sum_{j=p+1}^{n} \sum_{i=j-p-1}^{j} \beta_{n-j, j-i} y_{i} \\
= & \sum_{i=0}^{p} \sum_{j=p+1}^{i+p+1} \beta_{n-j, j-i} y_{i} \\
& +\sum_{i=p+1}^{n-p-1} \sum_{j=1}^{i+p+1} \beta_{n-j, j-i} y_{i}+\sum_{i=n-p}^{n} \sum_{j=i}^{n} \beta_{n-j, j-i} y_{i}
\end{aligned}
$$

where the last sum is the result of interchanging the order of summation. The elements of $W$ are now quite apparent from equation (3.2) by taking $i=n(-1) p+1$ in the second line. Thus, we have

$$
\begin{aligned}
g(W, z)= & \sum_{i=0}^{\infty} w_{i, 0} z^{i}=\beta_{0,0}+\left(\beta_{1,0}+\beta_{0,1}\right) z+\cdots+\left(\beta_{p, 0}+\cdots+\beta_{0, p}\right) z^{p} \\
& +\left(\beta_{p+1,0}+\cdots+\beta_{0, p+1}\right) z^{p+1}+\cdots
\end{aligned}
$$

noting that all subsequent terms are the sum of $p+2 \beta$ 's. Rearranging, we finally obtain

$$
g(W, z)=\sum_{i=0}^{\infty} \sigma_{i}^{*}(z) z^{i}
$$

in view of equation (3.1b). Also, the generating function for $W^{-1}$ is

$$
g\left(W^{-1}, z\right)=\{g(W, z)\}^{-1}=\left\{\sigma_{0}^{*}(z)\left[\sum_{i=0}^{\infty} \frac{\sigma_{i}^{*}(z)}{\sigma_{0}^{*}(z)} z^{i}\right]\right\}^{-1}
$$

Since $\sigma_{0}^{*}(z)$ has no zeros in $|z|<1$ and only simple zeros on $|z|=$ 1 (since $\sigma_{0}(z)$ is a simple von Neumann polynomial), $g\left(W^{-1}, z\right)$ is absolutely convergent in $|z|<1$ and hence $g(W, z)$ is likewise. The uniform bounds on $W_{N}$ and $W_{N}^{-1}$ then follow [15, theorem 4.3].

Remark. When choosing the $p+2$ coefficients of $\sigma_{0}(z)(j=n$ in lemma 2), one must relax the degree, $q_{n}=r-1 \leq p+1$, in order 
to make $\sigma_{0}(z)$ a simple von Neumann polynomial. In general, for a given $\alpha$, one can choose the $p+2-r$ free parameters so that $\sigma_{0}(z)$ has as many equal real roots as possible; see also an algorithm to achieve this in Gladwin $[\mathbf{9}, \mathbf{1 0}]$. In section 5 a family of methods for $r=3$ and $p=2$ and for all $\alpha \in[0,1)$ has been constructed but this quickly becomes prohibitive for large $r$ and $p$.

The next theorem summarizes the conditions for convergence of the discretization error, $e_{n}=y_{n}-y\left(x_{n}\right)$, as well as shows the minor modifications involved for a general kernel $K \in C^{\infty}\left(S_{2}\right)$.

Theorem 2. For the method defined by (2.2) we assume

(i) $K$ and $f$ satisfy the conditions of Theorem 1 and $K$ is sufficiently smooth on $S_{2}$

In addition, $f^{(j)}(0)=0, j=0,1, \ldots, r$ (where $r$ is the degree of smoothness),

(ii) the starting errors $e_{n}: n=0(1) p-1$ are of order $r$,

(iii) $\gamma_{n, i}$ and $\beta_{(n-j) i}$ are determined as in lemmas one and two with $q_{j}=p+1, p+1 \leq j \leq n-1$ and $\gamma_{p p} \neq 0$

(iv) $\beta_{0, i}$ are determined as in lemma two with $q_{n}=r-1 \leq p+1$ and the polynomial $\sigma_{0}(z)$ is simple Von Neumann and $\beta_{0,0} \neq 0$. Then there exists a positive finite constant $C$ such that $\left|e_{n}\right| \leq C h^{r}$ for $n=0(1) N$.

Proof. By using backward differencing on the error equation, corresponding to equation (2.2) and observing Taylor's formula for $K(x, t)$ (see Gladwin [8] for details) we may write

$$
\sum_{i=0}^{p+1} \beta_{0, i} e_{n-i}=K_{n n}^{-1}\left(h \sum_{i=0}^{n-1} \tilde{A}_{n, i} e_{i}+h^{\alpha-1}\left(E_{n}-E_{n-1}\right)\right)
$$

where i) $K_{n n}=K\left(x_{n}, x_{n}\right) \neq 0$ for all $n$ (this condition is needed for a unique continuous solution of equation (1.1))

ii) $\left\{\tilde{A}_{n, i}\right\}$ depends linearly on $\left\{\gamma_{n, i}\right\}$ and $\left\{\beta_{(n-j) i}\right\}, p+1 \leq j \leq n-1$, and $K\left(x_{n}, x_{j-i}\right)$, and

iii) $E_{n}=E_{n, p}+\sum_{j=p+1}^{n} E_{n, j}$

We note $E_{n}-E_{n-1}=O\left(h^{r+1-\alpha}\right)$ in view of the first part of assumption four, and by virtue of the root condition in the second part, the left 
hand side of equation (3.4) can be rewritten to depend only on $e_{n}$ and $\left\{e_{j}\right\}, j=0(1) p-1$, the starting errors, which are $O\left(h^{r}\right)$. The theorem follows with the application of a standard recursive inequality.

Remark. It should be noted that the factor $h$ before the summation in (3.4) arises from the differencing of the kernel $K\left(x_{n}, x_{i}\right)$ and subsequent Taylor's series application only.

From now on, we shall refer to $\sigma_{0}(z)$ as $\sigma(z)$ for ease of notation.

Remark. For the case $p=1$ and $r=3$ it is to be noted that

$$
\sigma(z)=\frac{\left(\frac{1}{2}(5-\alpha) z^{2}+(4-\alpha)(1-\alpha) z-\frac{1}{2}(1-\alpha)\right)}{(1-\alpha)(2-\alpha)(3-\alpha)}
$$

and is simple von Neumann for $0.44=(5-\sqrt{17}) / 2<\alpha<1$ (see Coppel $[4])$.

4. Numerical results. In this section Table 1 displays some numerical results which have been obtained using an equation of the form

$$
y_{n}=\frac{h^{\alpha-1} f\left(x_{n}\right)-\sum_{i=0}^{p} \gamma_{n, i} K\left(x_{n}, x_{i}\right) y_{i}-\sum_{i=0}^{n-1} w_{n, i} K\left(x_{n}, x_{i}\right) y_{i}}{w_{n n} K\left(x_{n}, x_{n}\right)}
$$

where $n=2(1) N, p=1, r=3$, and $y_{0}=y(0)$. An approximation for $y_{1}$ is obtained by solving

$$
\gamma_{10} K\left(x_{1}, x_{0}\right) y_{0}+\gamma_{11} K\left(x_{1}, x_{1}\right) y_{1}=h^{\alpha-1} f\left(x_{1}\right)
$$

where $\gamma_{10}$ and $\gamma_{11}$ are determined using the system (4.1) with $n=1$. For the main part of the method, the weights are determined using

$$
\sum_{i=0}^{p} \gamma_{n i}(n-i)^{k}=\frac{n^{k+1-\alpha}-(n-p)^{k+1-\alpha}}{k+1-\alpha}, \quad k=0(1) p
$$

$\sum_{i=0}^{p+1} \beta_{n-(p+1), i}(n-(p+1)+i)^{k}=\frac{[n-(p+1)+1]^{k+1-\alpha}-[n-(p+1)]^{k+1-\alpha}}{k+1-\alpha}$,

$$
k=0(1) p+1
$$


and

$$
w_{n, i}=\beta_{n-(p+1), p+1-i}+\left(1-\delta_{i}\right) w_{n-1, i-1}
$$

where $\delta_{0}=1$ and $\delta_{i}=0, i>0$. The example was solved using this method with $\alpha=.5$. The program was written in Fortran using double precision and run on the University's IBM 3091 computer. In particular, note that $y(0)=\lim _{x \rightarrow 0}(1-\alpha) f(x) x^{\alpha-1} / K(0,0)=0$ for the example below.

\section{Example.}

$$
\int_{0}^{x} \frac{e^{x} t y(t) d t}{(x-t)^{\alpha}}=\frac{e^{x} 5 ! x^{6-\alpha}}{(6-\alpha)(5-\alpha) \cdots(1-\alpha)}, \quad 0 \leq x \leq 5, \alpha=.5
$$

(Exact solution: $y(x)=x^{4}$.)

\begin{tabular}{|c|c|c|}
\hline & $h=.05$ & $h=.10$ \\
\hline$x_{n}$ & $\left|y_{n}-y\left(x_{n}\right)\right|$ & $\mid y_{n}-y\left(x_{n}\right)$ \\
\hline 1 & $2.82 \times 10^{-4}$ & $2.10 \times 10^{-3}$ \\
\hline 2 & $5.87 \times 10^{-4}$ & $4.52 \times 10^{-3}$ \\
\hline 3 & $8.94 \times 10^{-4}$ & $6.95 \times 10^{-3}$ \\
\hline 4 & $1.20 \times 10^{-3}$ & $9.40 \times 10^{-3}$ \\
\hline 5 & $1.51 \times 10^{-3}$ & $1.19 \times 10^{-2}$ \\
\hline
\end{tabular}

TABLE 1

5. Conclusions. We note that the example is the Abel equation for the case $\alpha=1 / 2$. This arises in various physical applications, Piessens and Verbaeten [16], for example.

To construct other convergent methods of order $r=3$ for all $\alpha \in$ $[0,1)$, one can take $p=2$ in Theorem two. This makes the coefficients of $\sigma(z)$ depend on a single parameter, $\beta_{0,0}$ say. Again using stability conditions as in Coppel [4], we can make $\sigma(z)$ simple von Neumann for

$$
\beta_{0,0} \in(L(\alpha), U(\alpha)), \quad \alpha \in[0,1)
$$


where

$$
L(\alpha)=\frac{1}{8}\left\{\frac{(4-\alpha)(1+\alpha)}{(1-\alpha)(2-\alpha)(3-\alpha)}-\frac{4-\alpha}{(1-\alpha)(2-\alpha)}+\frac{1}{1-\alpha}\right\}
$$

and

$$
U(\alpha)=L(\alpha)+\frac{1}{8} \frac{(4-\alpha)^{2}(1+\alpha)}{(1-\alpha)(2-\alpha)^{2}(3-\alpha)}
$$

\section{REFERENCES}

1. H. Brunner, Global solution of the generalized Abel integral equation by implicit interpolation, Math. Comp. 28 (1974), 61-67.

2. H. Brunner and M.D. Evans, Piecewise polynomial collocation for Volterra type integral equations of the second kind, J. Inst. Math. Appl. 20 (1977), 415-423.

3. R.F. Cameron and S. McKee, The analysis of product integration methods for Abel's equation using discrete fractional differentiation, IMA J. Num. Anal. 5 (1985), 339-353.

4. W.A. Coppel, Stability and asymptotic behaviour of differential equations, Heath Math. Monographs, Boston, 1965.

5. P.P.B. Eggermont, A new analysis of the trapezoidal-discretisation method for the numerical solution of the Abel-type integral equations, J. Integral Equations 3 (1981), 317-332.

6. L.E. Garey, The numerical solution of Volterra integral equations with singular kernels, BIT 14 (1974), 33-39.

7. - Numerical methods for second kind Volterra equations with singular kernels, Proc. Fourth Manitoba Conf. on Numer. Math. (1974), 253-263.

8. C.J. Gladwin, Quadrature rule methods for Volterra integral equations of the first kind, Math. Comp. 33 (1979), 705-716.

9. - On optimal integration methods for Volterra integral equations of the first kind, Math. Comp. 39 (1982), 511-518.

10. - An algorithm for the construction of optimal methods for the numerical solution of Volterra integral equations of the first kind, Math. Comp. 40 (1987), 625-632.

11. F. DeHoog and R. Weiss, Higher order methods for a class of Volterra integral equations with weakly singular kernels, SIAM J. Numer. Anal. 11 (1974), 1166-1180.

12. P. Linz, Numerical methods for Volterra integral equations with singular kernels, SIAM J. Numer. Anal. 6 (1969), 365-374.

13. - Product integration methods for Volterra integral equations of the first kind, BIT 11 (1971), 413-421.

14. C.H. Lubich, Fractional linear multi-step methods for Abel-Volterra integral equations of the first kind, IMA J. Numer. Anal. 7 (1987), 97-106.

15. S. McKee, Discretisation methods and block isoclinal matrices, IMA J. Num. Anal. 3 (1983), 467-491. 
16. R. Piessens and P. Verbaeten, Numerical solution of the Abel integral equation, BIT 13 (1973), 451-457.

17. W. Pogorzelski, Integral equations and their applications, Vol. 1, Pergamon Press, Oxford, 1966.

18. R. Weiss, Product integration for the generalized Abel equation, Math. Comp. 26 (1972), 177-190.

19. R. Weiss and R.S. Anderssen, A product integration method for a class of singular first kind Volterra equations, Num. Math. 18 (1972), 442-456.

Division of Mathematics, Engineering and Computer Science, University of New Brunswick, P.O. Box 5050, Saint John, NB E2L 4L5 